\title{
ASSOCIAÇÕES ENTRE SUPORTE FAMILIAR E SAÚDE MENTAL
}

\author{
A ssociations between family support and mental health
}

\author{
Mayra Silva de Souza ${ }^{a}$, Makilim Nunes Baptista ${ }^{b}$ \\ a Mayra Silva de Souza: D outoranda em Psicologia pela Universidade São Francisco - USF. Bolsista CAPE S Itatiba, SP - Brasil, \\ e-mail: souza.mayra@gmail.com

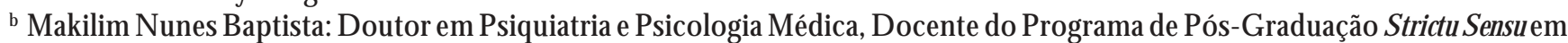 \\ Psicologia da Universidade São Francisco - USF. Bolsista produtividade CNPq. Itatiba, SP - Brasil, e-mail: \\ makilim.baptista@saofrancisco.edu.br
}

\section{Resumo}

A saúde é um estado de completo bem-estar físico, mental e social, e não consiste apenas em ausência de doença ou enfermidade. A saúde mental constitui-se como parte indispensável da saúde geral, permitindo ao indivíduo o aproveitamento pleno de suas capacidades cognitivas, relacionais e afetivas, 0 enfrentamento de dificuldades na vida, a produção no trabalho e a contribuição para ações em sociedade. O s critérios para saúde e doença mental não podem ser considerados independentemente, restritos ao indivíduo, uma vez que sua identidade a todo momento reflete uma experiência grupal. A saúde mental resulta do bom funcionamento interno do indivíduo, bem como sua capacidade de estabelecer ótimas relações com pessoas, sociedade e família. No grupo familiar, é possivel que haja uma predisposição à enfermidade, acarretando regressão, desintegração e ruptura na comunicação, porém, quando as funções familiares primordiais são cumpridas, a possibilidade existente é a de um potencial de promoção à saúde. 0 suporte familiar pode ser compreendido como manifestação de atenção, carinho, diálogo, liberdade, proximidade afetiva, autonomia e independência existente entre os integrantes da família e pode ser pensado como agente de proteção frente ao risco a doenças mentais, e agente amortecedor frente aos eventos estressantes, o que sustenta a criação de programas de prevenção e tratamento, visando ao restabelecimento da saúde.

Palavras-chave: Suporte familiar; Família; Saúde mental. 


\begin{abstract}
Health is a complete state of physical, mental and social well-being, not consisting only in the absence of illness or disease. Mental health can be considered an essential part of general health, allowing the individual full use of his cognitive, relational and affective abilities, coping strategies, work production and contributions to actions in society. The criteria for health and mental illness cannot be considered independent ones, restricted to the individual, once his identity, in all moments, reflects a group experience. Good health is a result of intern good function and the ability to establish great relations to people, society and family as well. In the family group, the existence of a tendency to illnesses is possible, resulting in communication's regression, disintegration and tearing, however, when primary family functions are fulfilled, the existent possibility is of a potential to health promotion. Family support may be understood as attention, care, dialog, freedom, affective proximity, autonomy and independence manifestations occurring among family members and it can be thought as a protective agent towards mental health's risks and also, a softener agent facing stress events, what can support the creation of prevention and treatment programs, considering health's reestablishment.
\end{abstract}

Keywords: Family support; Family; Mental health.

\section{INTRODUÇÃO}

A saúde mental é um conceito de difícil definição, pois diferencia-se de acordo com cada cultura, em um dado momento histórico, em uma determinada população, o que servirá de modelo para se considerar o que é um comportamento normal (conduta seguida pela maioria das pessoas que caracterizam essa população) e um comportamento desviante (como indica o próprio nome, conduta explicada pela minoria das pessoas que destacam-se por diferir das ditas "normais"). (Loureiro, 2000; Pasquali, Gouveia, Andriola, Miranda \& Ramos, 1996). Nota-se aqui, a influência do aspecto cultural e histórico na consideração dos estados de saúde e doença mental.

Ainda que seja difícil a conceituação da saúde mental, vários outros aspectos se veem imbricados e indispensáveis na determinação do estado de saúde de um indivíduo.Assim, éimportante considerar o aspecto biológico, no que tange a uma predisposição genética e hereditária do indivíduo para a doença mental, e também o aspecto sóciopsicológico, no que se refere à qualidade das relações que 0 indivíduo estabelece com grupos, que pode contribuir tanto para a saúde quanto para a doença mental. (Basic Behavioral Science Task Force of the National Advisory Mental Health Council, 1996).

As relações mais profundas e duradouras são estabelecidas dentro da família, principalmente nos primeiros anos de vida. É nela que um indivíduo estabelece seus primeiros vínculos de uma convivência grupal. A família é que vai mediar a relação do indivíduo com o mundo, provendo apoio afetivo e material (Ackerman, 1986; Campos, 2004; Ceberio, 2006). Q uando a família consegue oferecer a seus membros cuidado, carinho, atenção, diálogo, autonomia, empatia, afetividade, aceitação e liberdade, ela passa a funcionar como uma importante fonte suporte, indispensável ao desenvolvimento saudável de seus integrantes.

\section{H istórico e evolucão de conceito de doença e saúde mental}

0 conceito de doença mental vem evoluindo desde as sociedades primitivas, nas quais a causa da manifestação de tal doença era atribuída ao sobrenatural. Antigos egípcios, hebreus e árabes acreditavam que manifestações como essas traduziam a possessão de maus espíritos, demônios e deuses irados (Ribeiro, 1996, Vietta, Kodato \& Furlan, 2001).

Hipócrates, considerado o pai da medicina moderna, introduziu explicações fisiológicas que negavam essa abordagem sobrenatural como justificativa para o comportamento anormal. Suas explicações baseavam-se na noção de equilíbriodesequilíbrio entre substâncias, sistemas e órgãos, nas quais o comportamento era resultante da alteração dos níveis de quatro humores (bile negra, bile amarela, fleuma e sangue). 0 desequilíbrio de 
tais substâncias resultava em depressão, ansiedade e alterações bruscas de humor (Holmes, 1997).

$\mathrm{Na}$ Idade Média, e mais adiante no Renascimento, os doentes mentais não eram considerados apenas como possuídos pelo demônio, mas também como ameaça para a sociedade, devendo ser perseguidos e mortos. E ssa concepção deve-se à prevalência da religião cristã, que lançava mão da ideia de luta constante entre as forças do bem contra as forças do mal (Bastos, 1997).

As doenças mentais tornaram-se objeto de uma ciência que acabara de surgir, a psiquiatria. As explicações para as desordens de comportamento, afetividade e pensamento, mais uma vez pautavamse numa abordagem orgânica, estudada a partir de descobertas da neurofisiologia e da neuropsiquiatria. Mais tarde, as explicações para o comportamento anormal, concentraram-se em causas psicológicas e as várias abordagens da psicologia explicavam essa relação a sua maneira (Holmes, 1997, Ribeiro, 1996).

A saúde mental, para a Organização Mundial de Saúde (2000) é o estado que permite ao indivíduo o aproveitamento total de suas capacidades cognitivas, afetivas e relacionais, o enfrentamento de dificuldades na vida, a contribuição para ações em sociedade e a produção no trabalho. A partir desse conceito fica explícito o caráter transdisciplinar da saúde mental, uma vez que esta é influenciada por várias dimensões, inclusive a dimensão social. Sendo assim, os critérios para a saúde e doença mental não podem serconsideradosindependentemente, restritos ao indivíduo, uma vez que sua identidade, a todo momento, reflete uma experiência grupal. A saúde mental resulta do bom funcionamento psíquico interno do indivíduo, bem como sua capacidade de estabelecer relações adequadas com pessoas, sociedade e família. (Ackerman,1986; Ramos, 2002).

\section{Família e suporte familiar}

A família é entendida como rede primária deinteração social eprovedoradeapoio indispensável à manutenção da integridade física e psicológica do indivíduo. Assim, a família torna-se referência nas crenças, valores e comportamentos do indivíduo, à medidaquepune ou premiasuas atitudes, orientando0 quanto à forma de agir (Campos, 2004).

D eacordo com Cobb (1976), o suporte tem início desde o início do ciclo vital, é melhor manifestado nas primeiras relações estabelecidas com a mãe e é demonstrado de várias maneiras, porém, especialmente da maneira que o bebê é apoiado. Com o passar do tempo, 0 suporte se expande, se originando cada vez de outros membros da família, daí então dos colegas de trabalho e da comunidade, e talvez, em caso de necessidade especial, de um membro cuja profissão é de ajuda humana. À medida que o fim da vida se aproxima, o apoio social, em determinadas culturas, outra vez se origina, principalmente de membros da família.

A primeira e essencial função psíquica da família é dispensar afeto, fundamental à sobrevivência emocional dos recém-nascidos. Transmitir as experiências individuais e grupais acumuladas é uma tarefa psicológica e social, na medida em que os pais colaboram com a formação da identidade pessoal de seus filhos. Cabe ainda à família o fornecimento de um ambiente adequado para a aprendizagem empírica, assim como a mediação de informações com o universo circunjacente (O sório, 1996). É fundamental na dinâmica familiar a comunicação congruente, direcional, funcional e com carga emocional; regras coerentes e flexíveis; liderança compartilhada com filhos de forma democrática; autoestima; relação conjugal integrada, porém de forma que possibilite à família atuar como um todo, preservando a individualidade de cadaum (Féres-Carneiro, 1992).

Ao cumprir essas funções e sendo percebida como afetuosa, coesa, com boa comunicação, com regras flexíveis, mas com limites e fronteiras claras, a família consegue fornecer a seus membros, instrumentos fundamentais ao crescimento individual e pode ser entendida como um sistema de suporte (Lidchi \& Eisenstein, 2004). 0 suporte familiar é traduzido como 0 cumprimento de determinadas funções da família, tais como: coletar e disseminar informações sobre o mundo, transmitir ideologias, ajudar na formação de identidades, oferecer serviços práticos de ajuda concreta, apoio emocional, orientação e feedbadk e ainda guiar e mediar na solução de problemas, servindo de refúgio para repouso e recuperação de seus membros (Caplan, 1976 apud Campos, 2004).

Para Parker, Tupling e Brown (1979) 0 suporte familiar se traduz na quantidade de cuidados eproteção que os pais delegam a seusfilhos, incluindo comportamentos de afeto, cooperação, sensibilidade, aceitação, indiferença, rigor, rejeição, punição, controle, superproteção, estímulo à autonomia e à independência. Procidano e Heller (1983) apontam 
uma compreensão na qual o suporte é entendido como grau de satisfação pela família diante das necessidades de informação, apoio e feedbadk. Para Moos(1990), suportefamiliarinclui grau de confiança, afeto, apoio e ajuda entre os membros da família.

Suporte familiar pode ser entendido como a capacidade da família em oferecer a seus membros: constância, cuidado, carinho, atenção, diálogo, informação, autonomia, empatia, afetividade, aceitação e liberdade (Baptista \& O liveira, 2004). Sendo assim, esse conceito refere-se às características psicológicas que a família oferece a seu membro, o que se diferencia do conceito de estrutura familiar, que se refere às características físicas tais como o número de pessoas pertencentes a uma família, sua disposição e composição. De acordo com os estudos de McFarlene, Bellissimo e Norman (1995), não há relação direta entre estrutura familiar e suporte familiar, uma vez que as diferentes estruturas familiares têm capacidade de oferecer suporte familiar adequado.

De acordo com Campos (2004), o efeito principal do suporte fundamenta-se à medida que é percebido pelo receptorcomo satisfatório, demaneira que esse receptor sinta-se valorizado, amado, reconhecido, compreendido, cuidado e protegido e, ainda, fazendo parte de uma rede de informações e recursos que com ele são partilhados. É essa percepção que vai permitir ao indivíduo enfrentar 0 ambiente, de forma a lhe trazer resultados positivos que contribuam para o seu bem-estar psicológico, aumento da autoestima e redução do estresse.

\section{Suporte familiar e saúde mental}

As relações estabelecidas na família parecem constituir uma fonte de relações que contribui nos processos de saúde ou doença. No agrupamento familiar é possível que haja uma predisposição à enfermidade, ocasionando desintegração, regressão e ruptura na comunicação, porém, quando as funções primordiais da família são desempenhadas, tais como afeição, proteção, formação social, autonomia, etc., a possibilidade existente é a de um potencial de promoção à saúde (Féres-Carneiro, 1996). Segundo O sório (1996), 0 oferecimento de um adequado suporte pelo grupo familiar favorece a superação das crises vitais, ou melhor, da desestruturação causada por essas crises.
A família, ao mesmo tempo em que é vista como a mais importante fonte de suporte, é também concebida como fonte mais importante de estresse para seus membros, afetando de maneira poderosa nos processos de saúde e doença. $\mathrm{Na}$ família, 0 suporte se estrutura de forma mais consistente, pois énelaqueosvínculostornam-seestáveis eduradouros e, talvez por isso, as crises que afetam 0 funcionamento familiar são estressantes. 0 estresse vivenciado na família está ligado ao comportamento, à necessidade e à personalidade de cada membro da família, o que gera impacto na interação com os outros membros do sistema familiar, algumas vezes produzindo estresse. O s conflitos entre os membros da família podem vir de comportamentos imprudentes, problemas financeiros e de objetivos contrários (Campos, 2004).

Segundo Féres-Carneiro (1992), a configuração da dinâmica familiar, cujo funcionamento pode ser facilitador ou dificultador, traduz a formação da saúde mental dos membros de uma família. ParaZ amberlan e Biasoli-Alves (1996), a promoção de um saudável e adequado desenvolvimento da criança está intimamente relacionada com a qualidade das relações e interações constituídas entre os membros da família. De acordo com Steinhausen (1985), mudanças no funcionamento da família, no que tange às práticas inadequadas de atenção às crianças, estão relacionadas com a gravidade dos distúrbiosinfantis.

0 provimento e o recebimento do suporte familiar influi diretamente no bem-estar físico, psíquico e social do indivíduo, sendo que a falta desse suporte é um dos fatores que traduz predisposições à doença mental. A percepção e 0 recebimento de suporte pelos membros da família, constituem fontes fundamentais para a manutenção da saúde mental, no que tange à promoção de benefícios nos processos fisiológicos (sistema endócrino, cardiovascular e imunológico), ao enfrentamento de situações estressantes, e no alívio dos estresses físico e mental. (Basic Behavioral Science Task Force of the National Advisory Mental Health Council, 1996; Uchino, Cacioppo e KiecoltGlaser, 1996).

O relacionamento que os pais ou cuidadores estabelecem com a criança durante a infância é de suma importância. $\mathrm{O}$ afeto, a atenção e o cuidado constante dispensado permite que a criança se desenvolva normalmente. Já a não- 
transmissão desses cuidados pode aumentar a probabilidade dessa criança manifestar distúrbios mentais e comportamentais, tanto duranteainfância quanto em fases posteriores da vida (Organização Mundial da Saúde, 2001).

Evidências científicas indicam que altos níveis de controle, especialmente a afirmação do poder (demandas acompanhadas por frequente punição física), podem ter consequências prejudiciais para a criança. Assim, a concepção de controle tem sido distinguida de duas formas: a primeira forma de controle proporciona estrutura ao indivíduo, baseia-se em padrões racionais para 0 comportamento infantil, envolvendo comunicação e encorajando a criança a se desenvolver, ser independente, cooperar e conviver em sociedade; a segunda forma combina rigidez com cumprimento de regras fixas com pobre fundamento, desencorajando o desenvolvimento de atributos positivos na criança (Basic Behavioral Science Task Force of the National Advisory Mental Health Council, 1996).

Parker, Tupling e Brown (1979) postularam que os pais que dispensam afeto e são empáticos permitem que 0 filho tenha uma maior autoestima e isso proporciona proteção contra a depressão na fase adulta. Os autores explicaram que a superproteção está relacionada à depressão através da inibição da autonomia e da competência social. Ao desencorajar a independência e nutrir uma expectativa de que as consequências não dependem de respostas, 0 abandono e a depressão são providos quando são enfrentados os fatores de estresse.

Kashani, Canfield, Borduin, Soltys e Reid (1994) investigaram a relação da percepção familiar em crianças e o suporte social para o comportamento de desesperança destas. Participaram dessa pesquisa 100 crianças pacientes psiquiátricas que responderam uma série de medidas de autorelato, incluindo o Social Support Q uestionnaire-Self Report, a Scale of Independent Behavior, e a H opelessness Scale for $C$ hildren. Crianças com baixo nível desuportefamiliar e social mostraram-se mais desatentas, ofensivas e não-cooperativas. Em adição, crianças que relataram menos pessoas suportivas em suas vidas apresentaram alto nível de desesperança. Esses dados enfatizam a conexão entre sistemas desuporte social, psicopatologia e desesperança. Esses relacionamentos poderiam indicar que crianças necessitam sentir que estão sendo cuidadas e acolhidas por algum tipo de suporte, e que isso serviria então para diminuir as probabilidades destas desenvolverem sérios problemas psicopatológicos e sentimentos de desesperança.

Em um estudo de revisão, Baptista, Baptista e Dias (2001), estudaram a estrutura e 0 suporte familiar como fatores de risco para a depressão em adolescentes. Os autores ressaltam que não se pode pensar em uma causa específica para o desenvolvimento da depressão, que é multifacetária, sendo influenciada por fatores biológicos/genéticos, psicológicos e sociais. Porém, as rápidas mudanças sociais familiares, no que tange às mudanças na composição, estrutura física e, consequentemente, nas regras e papéis da família, acabam por colaborar com a prevalência de depressão na população adolescente. A família ainda deve ser considerada como amortecedora frente aos eventos estressantes, enfrentados no cotidiano de adolescentes.

O estudo deMcFarlene, Bellissimo eN orman (1995) avaliou a associação entre a estrutura familiar, a integração familiar e 0 bem-estar dos filhos na adolescência, estabelecendo o contraste de famílias intactas com aquelas que foram afetadas por alguma mudança. Foi feita uma pesquisa com 810 estudantes de 11 escolas de um único sistema educacional. Os resultados indicaram que a configuração familiar não foi o principal fator determinante na eficiência da parte funcional da família. Em vez disso, a maneira como os pais tratam os filhos foi considerado 0 fator determinante para a integração familiar e o bem-estar dos adolescentes. O s cuidados da mãe e do pai foram associados à integração familiar saudável ao passo que a superproteção foi negativamente associada a este resultado. As famílias nas quais houve morte de um dos pais pareceram funcionar (integrar-se) tão bem como as familias cujos pais estavam sempre presentes, mais uma vez ressaltando a ideia que a função familiar não é determinada pela estrutura. No entanto, não está bem estabelecida ainda a inexistência da associação entre saúde mental e estrutura familiar.

Bassuk, Mickelson, Bissel e Perloff (2002) investigaram o papel de vários membros da família, diferenciando os pais e os filhos, e descobriram que a relação com os irmãos proporcionou resultados positivos para a saúde mental (frequentemente mais forte que a relação com os pais). Em algumas famílias pobres que compuseram a amostra, as relações entre irmãos podem ter assumido um nível 
maior de importância por causa de rupturas na família de origem e a falta compreensível de uma criação consistente por parte dos pais, resultando no estresse inevitável associado à pobreza. Q uando os adultos são incapazes de fornecer proteção, segurança, e alimentação, os irmãos podem recorrer uns aos outros.

D eterminadas experiências infantis, como maus tratos emocionais por parte dos pais ou cuidadores, perda dos pais, seja por separação (divórcio) ou por morte de algum deles, podem resultar em prejuízos duradouros no desenvolvimento das crianças e adolescentes, comprometendo a vida adulta (Heim \& Nemeroff, 2001). Bemporad e Romano (1993), em uma revisão acerca das experiências infantis e depressão na vida adulta, encontraram um alto grau de disfunções nas histórias infantis de pacientes deprimidos, quando comparados aos controles. Nesse estudo, a depressão adulta relacionou-se com mau tratamento na infância, como rejeição e falta de afeto.

Em uma meta-análise, que reuniu dados de 2432 sujeitos, foi encontrada uma associação significativamente alta entre a perda de um dos pais com indivíduos que sofriam de depressão, quando comparados aos que não sofriam de depressão. A perda de um dos pais antes dos 11 anos relacionouse com mulheres deprimidas; e o fato de ter perdido a mãe mais cedo ainda poderia representar um risco aumentado para a depressão. (Patten, 1991).

Bron, Strack e Rudolph (1991) estudaram 328 pacientes com depressão, desordem distímica ou desordem de ajuste com humor deprimido. Eles foram questionados sobreperdas sofridas nainfância por morte de um ou ambos os pai e também por divórcio dos pais com pelo menos um ano de separação. Não houve estatísticas significativas entre experiências de perda na infância e tipo de depressão, sexo e idade. Contudo, foi encontrado um aumento na incidência de tentativas de suicídio naqueles pacientes com experiências de perdas na infância, por separação dos pais e por morte de um deles, sendo 0 aumento da tendência suicida atribuído principalmente à perda do pai.

Landerman, George e Blazer (1991) investigaram a vulnerabilidade de adultos para desordens psiquiátricas diante da separação dos pais antes dos 10 anos de vida, devido a divórcio ou morte e diante da presença de doença mental nos pais durante os primeiros anos da infância. Os dados obtidos foram de uma amostra estratificada composta por 3801 adultos. Os resultados sugerem que ter um dos pais com doença mental aumenta a probabilidade dos eventos de vida estressantes resultarem em depressão, porém, ainda não se sabe a que se deve esse aumento (fatores genéticos ou ambientais) . A separação por divórcio dos pais, associadacom eventos devida estressantes, apontam para 0 aumento da vulnerabilidade para problemas psiquiátricos e alcoolismo. E a separação por morte de um dos pais não interage com eventos de vida estressantes no sentido de afetar a probabilidade de problemas psiquiátricos, ao menos nesse estudo.

McLeod (1991) concluiu que o evento da perda de um dos pais por divórcio estava mais fortementerelacionado com consequências negativas na vida adulta do que a perda por morte. E as correlações dos eventos de perda edas consequências negativas eram mais fortes nas mulheres do que nos homens. A morte de um cônjuge pode aumentar a probabilidade do outro ficar doente ou morrer, ao menos durante dois anos. E os viúvos que não se casam novamente têm maiores taxas de mortalidade do que os que casam (Ramos, 2002).

Kendler, Neale, Keesler, Health e Eaves (1992), examinaram a relação entre perda dos pais antes dos 17 anos e psicopatologia na idade adulta. Essa relação variou de acordo com o tipo de perda (por separação conjugal ou morte dos pais) e de acordo com a forma de psicopatologia. A perda dos pais por separação conjugal associou-se principalmente com maior risco para depressão e ansiedade generalizada. Já a perda por morte da mãe especificadamente relacionou-se ao maior risco para transtorno do pânico e perda por morte dos pais, tanto morte materna como morte paterna, associouse a risco maior para fobias.

Em uma amostra japonesa composta por 122 pacientes internados com quadro de depressão unipolar e 94 que nunca estiveram deprimidos foi comparadaa ocorrência de perda dos pais por morte e por separação conjugal antes dos 17 anos de idade. A perda por morte materna foi significativamente mais comum em depressivos que no grupo controle. E a perda por separação dos pais teve tendência aumentada no grupo deprimido (Knugi, Sugawara, Aoki, Nanko, Hirose \& Kazamatsuri, 1995).

Luecken (2000) estudou a associação entre perda por morte de um dos pais, sentimentos de hostilidade, depressão, suporte social e qualidade das relações familiares em adolescentes. 
Participaram dessa pesquisa 30 estudantes universitários que experimentaram a morte de um dos pais antes dos 16 anos, e 31 estudantes em condição controle. Foram utilizados questionários para medir o suporte social (T heInterpersonal Support E valuation), a hostilidade (C ook Medley H ostility Scale), a depressão (Beck D epression Inventory) e a qualidade das relações familiares (Moos Family E nvironment Scale). Aqueles com perda de um dos pais apresentaram altos escores de hostilidade, maior intensidade de sintomas depressivos e menor suporte social apenas quando apresentavam pobres relações familiares atuais.

Souza (2007) estudou a relação entre 0 suportefamiliareasaúdemental em 520 universitários, utilizando o Inventário de Percepção de Suporte Familiar (IPSF) e o Questionário de Saúde G eral de Goldberg (QSG). Foram encontradas correlações significativas entre os instrumentos, o que demonstra que a percepção de demonstrações de afeto, carinho, expressões verbais e não-verbais, habilidade na resolução de situações-problema, sentimentos positivos, tais como inclusão e compreensão, além do estabelecimento de relações de confiança, liberdade e privacidade entre os membros da família, colaboram paramenorsintomatologiadeestressepsíquico , desejo de morte, desconfiança do próprio desempenho, distúrbios do sono, distúrbios psicossomáticos e severidade de doença mental.

\section{CONSIDERAÇÕES FINAIS}

Diante da literatura pesquisada nessa revisão, é possível destacar características importantes na associação entre o suporte familiar e a saúde mental, como a investigação da estrutura familiar, sexo, raça, curso, nível econômico. Outras variáveis ainda se mostram relacionadas, a saber: eventos de vida estressantes, estratégias de enfrentamento (coping), personalidade, resiliência, lócus de controle, autoeficácia, dentre outras. Todas essas variáveis podem diferenciar os sujeitos quanto à percepção do suporte familiar e quanto ao estado de saúde mental destes, e por isso mostram-se relevantes no estudo com os construtos envolvidos.

0 estudo da associação entre suporte familiare saúde mental fundamenta-se na crescente importância que vários autores têm dado ao fato de que as relações sociais podem ter um papel essencial para manter ou mesmo promover a saúde mental (Ackerman, 1986; Baptista, 2004; Basic Behavioral Science Task Force of the National Advisory Mental Health Council, 1996; Bassuk, Mickelson, Bissel, \& Perloff, 2002; Campos, 2004; Féres-Carneiro, 1992; Kashani, Canfield, Borduin, Soltys, \& Reid, 1994; O MS, 2001; Ramos, 2002; Souza, 2007). Pensando numa forma de intervenção para a saúde mental, programas que enfatizem o suporte da família podem atuar como agente preventivo e protetivo frente aos riscos para doenças mentais, o que sustenta a criação de programas de prevenção e tratamento, visando 0 restabelecimento da saúde.

\section{REFERÊNCIAS}

Ackerman, N. (1986). Diagnóstico e tratamento das relações familiares. Porto Alegre: Artes Médicas.

Baptista, M. N., Baptista, A. S. D., \& Dias, R. R. (2001). Estrutura e suporte familiar como fatores de risco na depressão de adolescentes. Psicologia Ciência e Profissão, 21(2), 52-61.

Baptista, M. N., \& Oliveira, A. A. (2004). Sintomatologia de depressão e suporte familiar em adolescentes: um estudo de correlação. Revista Brasileira de Crescimento e Desenvolvimento H umano, 14(3), 58-67.

Basic Behavioral Science Task Force of the National Advisory Mental Health Council. (1996). Basic behavioral science research for mental health: Family processes and social networks. American Psychologist, 51, 622-630.

Bassuk, E. L., Mickelson, K. D., Bissel, H. D. \& Perloff, J. N. (2002). Role of kin and nonkin support in the mental health of low-income women. American Journal of Orthopsychiatry, 72(1), 39-49.

Bastos, C. L. (1997). Introdução. In C. Lyra Bastos. Exame psíquico: U ma introdução prática à psicopatologia. (pp. 01-25). Rio de Janeiro: Revinter.

Bemporad, J. R., \& Romano, S. (1993). Childhood experience and adult depression: a review of studies. American Journal Psychoanalysis, 53, 301-315. 
Bron, B., Strack, M., \& Rudolph, G. (1991). Childhood experience of loss and suicide attempts: significance in depressive states of major depressed and dystimic or adjustment disorder patients. Journal Affective Disorders, 23, 165-72.

Campos, E. P. (2004). Suporte social e família. In J. Mello Filho. Doença e família. (pp. 141161). São Paulo: Casa do Psicólogo.

Ceberio, M. R. (2006). Viejas y nuevas famílias: La transición hacia nuevas estructuras familiares. Interpsiquis. Recuperado em 18 fev. 2006: www.psiquiatria.com

Cobb, S. (1976). Social support as a moderator of life stress. Psychosomatic Medicine, 38(5), 300-314.

Féres-Carneiro, T. (1992). Família e saúde mental. Psicologia: Teoria e Pesquisa, 8, 485-493.

Féres-Carneiro, T. (1996). Família: Diagnóstico e terapia. Petrópolis: Vozes.

Heim, C., \& Nemeroff, C. B. (2001). The role of childhood trauma in the neurobiology of mood and anxiety disorders: preclinical and clinical studies. Biological Psychiatry, 49, 1023-1039.

Holmes, D. S. (1997). História e definições. In S. D avid Holmes. Psicologia dos Transtornos Mentais (pp. 25-35). Porto Alegre: Artes Médicas.

Kashani, J. H., Canfield, L. A., Borduin, C. M., Soltys, S. M., \& Reid, J. C. (1994). Perceived family and social support: Impact on children. Journal American Academy of Child and Adolescent Psychiatry, 33(6), 819-823.

Kendler, K. S., Neale, M. C., Kessler, R. C., Heath, A. C., \& Eaves, L. J. (1992). Childhood parental loss and adult psychopathology in women: A twin study perspective. Archives General Psychiatry, 49, 109-16.

Kunugi, H., Sugawara, N., Aoki, H., Nanko, S., Hirose, T., \& Kazamatsuri, H. (1995). Early parental loss and depressive disorder in Japan. E uropean Archives Psychiatry Clinical Neuroscience, 245, 109-113.
Landerman R., G eorge, L. K., \& Blazer, D. G. (1991). Adult vulnerability for psychiatric disorders: interactive effects of negative childhood experiences and recent stress. Journal Nervouse Mental Disorders, 179, 656-663.

Lidchi, V., \& Eisenstein, E. (2004). Adolescentes e famílias no contexto médico. In J. Mello Filho. D oença e família. (pp. 217-231). São Paulo: Casa do Psicólogo.

Loureiro, S. R. (2000). Transtornos de personalidade e avaliação psicodiagnóstica. In F. F. Sisto, E. T. B. Sbardelini \& R. Primi (O rg.). Contextos e questões da avaliação psicológica. (pp. 51-61). São Paulo: Casa do Psicólogo.

Luecken, L. J. (2000). Attachment and loss experiences during childhood are associated with adult hostility, depression, and social support. Journal Psychosomatic Research, 49, 85-91.

McFarlene, A. H., Bellissimo, A., \& Norman, G. R. (1995). Family structure, family functioning and adolescent well-being: The transcendent influence of parental style. Journal of Child Psychology and Psychiatry, 36, 847-864.

McLeod, J. D. (1991). Childhood parental loss and adult depression. Journal Health Social Behavior, 32, 205-20.

Moos, R. H. (1990). D epressed outpatients's life contexts, amount of treatment and treatment outcome. Journal of Nervous and Mental Disease, 178, 105-112.

Organização Mundial de Saúde (O MS) (2000). Conselho Executivo - 1070 sessão. Recuperado em 21 mar. 2005, da Saúde Mental: www.saudemental.med.br/ O MS.htm

Organização Mundial de Saúde (O MS) (2001). Relatónio Mundial da Saúde: Saúde mental: Nova concepção, nova esperança. Recuperado em 20 de nov. 2005, da World health organization: www.who.int/ whr/ 2001/ en/whr01_po.pdf

O sório, L. C. (1996). O que é a família, afinal ? In L. C. O sório, Família hoje. (pp. 14-33). Porto Alegre: Artes Médicas. 
Parker, G., Tupling, H., \& Brown, L. B. (1979). A parental bonding instrument. British Journal of Medical Psychology, 52, 1-10.

Pasquali, L., Gouveia, V. V., Andriola, W. B., Miranda, F. J., \& Ramos, A. L. (1996). Q uestionário de sáude geral de Goldberg: Manual técnico. São Paulo: Casa do Psicólogo.

Patten, S. B. (1991). The loss of a parent during childhood as a risk factor for depression. Canadian Journal Psychiatry, 36, 706-11.

Procidano, M. E., \& Heller, K. (1983). Measures of perceived social support from friends and from family: Three validation studies. American Journal of Community Psychology, 11(1), 1-24.

Ramos, M. P. (2002). A poio social e saúde entre idosos. Sociologias, 7, 11-20.

Ribeiro, P. R. M. (1996). Saúde mental: Dimensão histórica e campos de atuação. São Paulo: EPU.

Souza, M. S. de (2007). Suporte familiar e saúde mental: Evidência de validade baseada na relação entre variáveis. D issertação de mestrado, programa de Pós-Graduação Stricto Sensu em Psicologia, Universidade São Francisco, Itatiba.
Steinhausen, H. C. (1985). Psychiatric disorders in children and family dysfunction. A study of migrant worker's families. Social Psychiatry, 20, 11-16.

Uchino, B. N., Cacioppo, J. T., \& Kiecolt-Glaser, J. K. (1996). The relationship between social support and physiological processes: A review with emphasis on underlying mechanisms and implications for health. Psychological Bulletin, 119, 488-531.

Vietta, E. P., Kodato, S., \& Furlan, R. (2001). Reflexões sobre a transição paradigmática em saúde mental. Revista Latino-Americana de Enfermagem, 9(2), 97-103.

Zamberlan, M. A. T., \& Biasoli-Alves, Z. M. N. (1996). Interações familiares: Teoria, pesquisa e subsídios à intervenção. Londrina: UEL.
Recebido: 11/ 08/ 2007

Received: 08/ 11/ 2007

Aprovado: 21/03/ 2008

A pproved: $03 / 21 / 2008$ 